
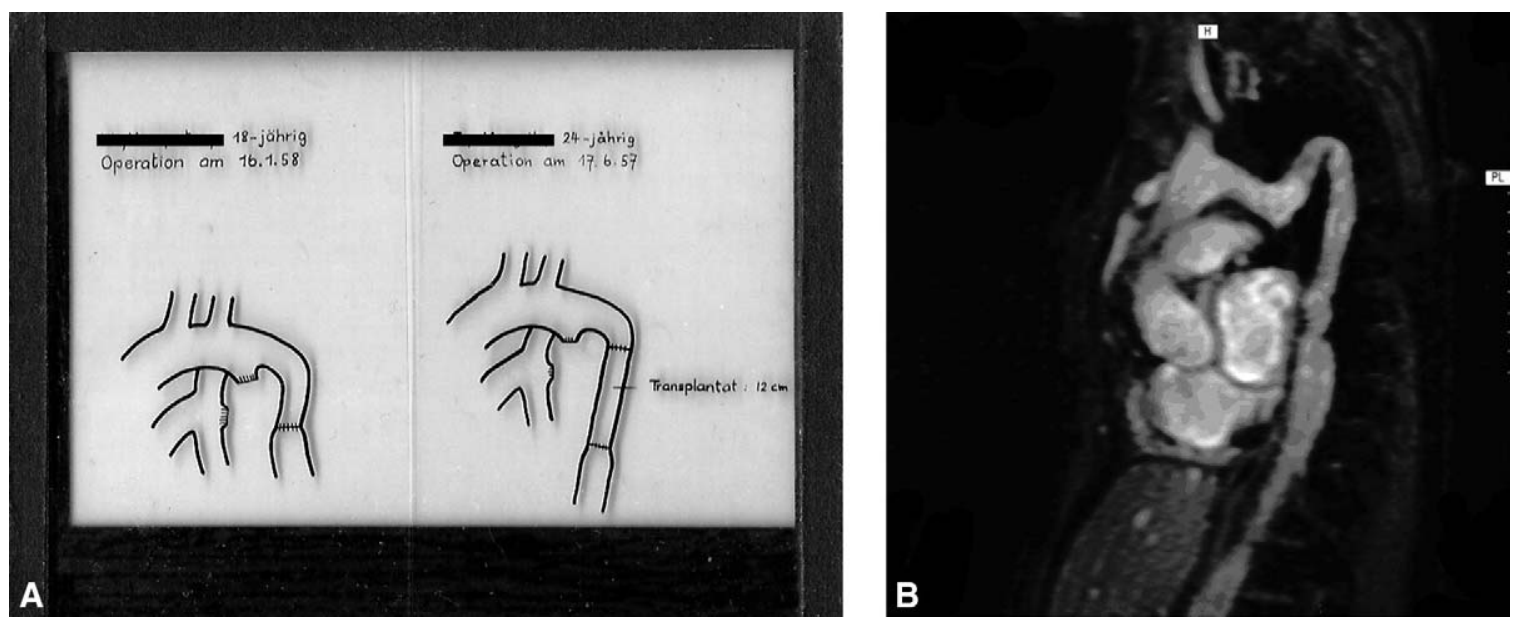

Figure 2. A, Original slide with Nissen's drawings showing technical procedure in general (right side of slide demonstrates situation in our patient). B, Computed tomographic scan, performed in 1992, shows calcification of replaced part of aorta. See also p. 969.

\title{
Repair of anomalous right and circumflex coronary arteries arising from the pulmonary artery
}

Giovanni Battista Luciani, MD, ${ }^{a}$ Fauzia Vendrametto, MD, ${ }^{\mathrm{b}}$ Luca Barozzi, MD, ${ }^{\text {a }}$ Rainer Oberhollenzer, MD, Walter Pitscheider, MD, ${ }^{\mathrm{b}}$ and Alessandro Mazzucco, MD, ${ }^{\mathrm{a}}$ Verona and Bolzano, Italy

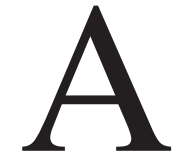

nomalous origin of the right coronary artery (RCA) from the pulmonary artery (PA; ARCAPA) is a rare congenital anomaly. In the last 40 years, 72 cases have been described, and 40 patients have undergone repair. ${ }^{1-3}$ Origin of the circumflex coronary artery (CxA) from the PA (ACCAPA) is even more unusual, with only 12 patients identified in the literature. ${ }^{4,5}$ Contrary to ARCAPA, anomalous origin of the CxA is frequently associated with other cardiac anomalies, although isolated ACCAPA has been reported. ${ }^{4,5}$ Different from the low mortality (5\%) associated with repair of the former anomaly, ${ }^{1-3}$ the risk of operation for ACCAPA appears

From the Division of Cardiac Surgery, University of Verona, ${ }^{a}$ Verona, Italy, and the Division of Cardiology, Bolzano City Hospital, ${ }^{\mathrm{b}}$ Bolzano, Italy.

Received for publication March 21, 2006; revisions received May 5, 2006; accepted for publication May 12, 2006.

Address for reprints: Giovanni Battista Luciani, MD, Division of Cardiac Surgery, University of Verona, O. C. M. Piazzale Stefani 1, Verona 37126, Italy (E-mail: gbluciani@yahoo.com).

J Thorac Cardiovasc Surg 2006;132:970-2

$0022-5223 / \$ 32.00$

Copyright $\odot 2006$ by The American Association for Thoracic Surgery doi:10.1016/j.jtcvs.2006.05.053 high $(29 \%) .{ }^{4}$ Here we describe a previously unreported congenital anomaly consisting of origin of both the RCA and CxA from the main PA successfully repaired by means of coronary reimplantation.

\section{Clinical Summary}

A 51-year-old man with an unremarkable clinical history had an incidental electrocardiographic (EKG) finding of inferior wall myocardial infarction. Treadmill exercise testing confirmed inferolateral ischemia, which was reversible on exercise Thallium-201 scintigraphic scanning. Preoperative echocardiographic examination showed normal left ventricular dimensions (enddiastolic diameter, $58 \mathrm{~mm}$; end-systolic diameter, 38), preserved function (ejection fraction, $58 \%$ ), and mild mitral regurgitation. Coronary angiography demonstrated a dilated and tortuous left anterior descending artery (Figure 1) freely connected with a dilated and tortuous RCA through the posterior descending artery, septal branches, and a mesh of thin epicardial vessels (Figure 1, A). The RCA drained into the pulmonary trunk. The CxA seemed to originate from the pulmonary trunk through the RCA (Figure 1,B). Considering the young age of the patient and the evidence of silent myocardial ischemia, repair was advised. After median sternotomy, origin of both the RCA and CxA from the right-facing pulmonary sinus through the common ostium was confirmed: the $\mathrm{CxA}$ coursed anterior to 


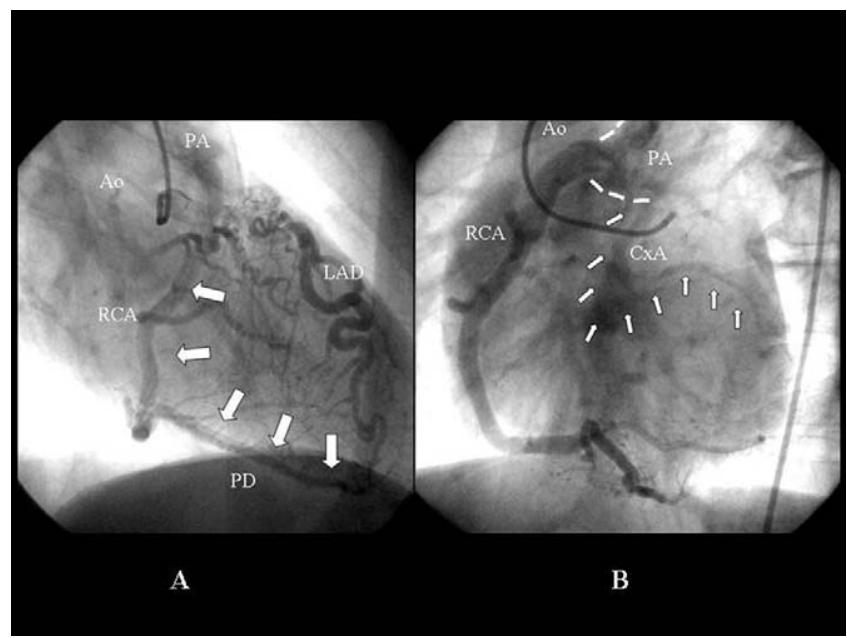

Figure 1. A, Right anterior oblique view late after left coronary artery angiography. The left anterior descending artery is widely connected through septal and posterior descending artery branches with a dilated right coronary artery $(R C A)$, which opacifies the pulmonary artery (PA). B, Left anterior oblique view late after left coronary artery angiography. Dye is still visible in the RCA, which partly contrasts the PA root (broken lines). A small branch originating from the RXA and corresponding to the circumflex artery (CXA) is also visible (arrows). Before reaching the obtuse margin of the heart, the course of the CxA is also anomalous. Ao, Aorta; $L A D$, left anterior descending artery; $P D$, posterior descending artery.

the pulmonary trunk, then leftward above the left atrial appendage to reach the obtuse margin of the heart (Figure 2, A). The proximal course of the RCA and CxA was carefully dissected and freely mobilized from the aortic and pulmonary roots. During normothermic cardiopulmonary bypass, the aorta and PA were crossclamped to avoid runoff of cardioplegia into the pulmonary root, and the heart was arrested. After transection of the PA below the bifurcation, the common coronary ostium was harvested with the entire sinus of Valsalva, and the defect on the pulmonary root was repaired with autologous pericardium (Figure 2, B). The aorta was incised $2 \mathrm{~cm}$ above the sinotubular junction, and the coronary button was reimplanted with a trapdoor technique to avoid coronary kinking. Postoperative recovery was uneventful, with patient discharge on postoperative day 7 . At 3 months' follow-up, EKG findings were comparable with preoperative results, and treadmill exercise testing was unremarkable.

\section{Discussion}

Although ARCAPA occurs more commonly (70\% of reported patients) as an isolated malformation, ${ }^{1}$ ACCAPA presents with associated congenital lesions in up to three quarters of cases. ${ }^{4}$ Here we describe an unusual case of isolated anomalous origin of both the RCA and CxA from the main PA. Indication to repair is well established in patients presenting with associated anomalies; how-

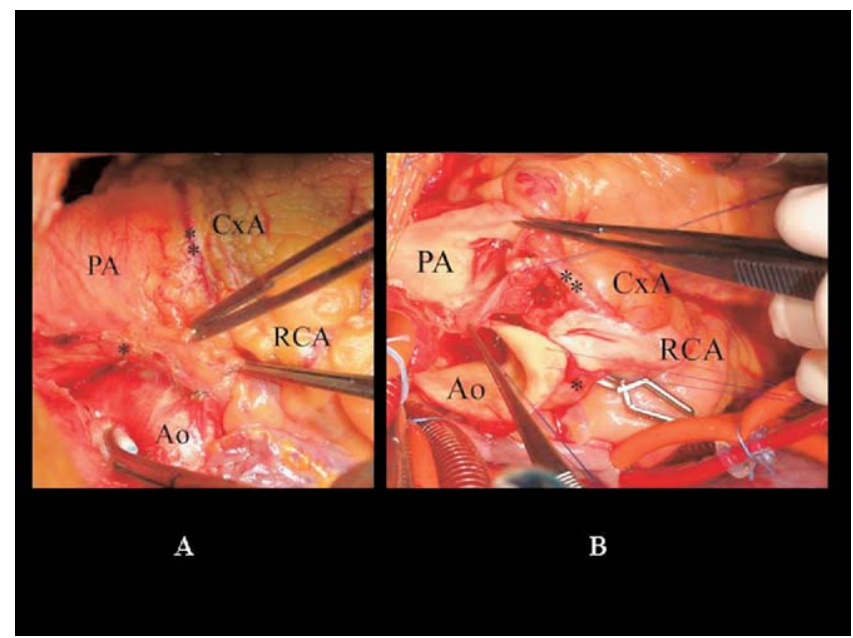

Figure 2. A, Operative view after dissection of the aortopulmonary recess. Origin of the right coronary artery (RCA) from the right anterior pulmonary sinus is clearly shown. Small collateral branches of the proximal RCA, giving rise to aortic vasa vasorum, have been sectioned (clips) to mobilize the coronary artery. The proximal course of the anomalous circumflex artery (CxA; double star) is also visible. B, Intermediate step of coronary artery reimplantation. The transected ascending aorta $(\mathrm{Ao})$ and pulmonary artery (PA) are visible. The defect in the coronary sinus of Valsalva of the PA has been patched with autologous pericardium (left-hand forceps). The anomalous coronary artery button, from which both the RCA (star) and the CxA (double star) arise, has been harvested.

ever, the need for surgical intervention has been debated in asymptomatic patients with isolated ARCAPA or ACCAPA. ${ }^{1,4}$ The fact that only $40(56 \%)$ of 72 patients with $\mathrm{ARCAPA}^{1-3}$ and $7(58 \%)$ of 12 patients with ACCAPA $^{4,5}$ have historically undergone operations highlights this controversy. Nonetheless, adverse events, including myocardial infarction, arrhythmias, left ventricular aneurysm, mitral insufficiency, cardiomyopathy, and even sudden death, have been described in patients with both types of anomalies. $^{1,4,5}$ Therefore, the tendency to advise surgical repair in asymptomatic patients has strengthened. Although isolated coronary anomalies that remain silent into adulthood are compensated by a network of collateral arteries, such adaptive mechanisms cannot be trusted indefinitely because of the combined effects of coronary to pulmonary steal and chronic left ventricular volume overload caused by left to right shunting. Accordingly, both resting EKG and inducible ischemic lesions were evident in our otherwise healthy patient. In addition, the rare combination of ARCAPA with ACCAPA provided stronger evidence in favor of repair. Technical options for treatment of anomalous coronary origins include simple ligation, bypass grafting, and reimplantation. The first 2 strategies have more commonly been reported in adult patients, whereas the latter is the preferred option in children and adolescents. ${ }^{1,4}$ The rationale is that collateral coronary circulation, well developed in adults, makes ligation with or without bypass grafting affordable, whereas translocation of coronary buttons 
might be more challenging. Recent clinical evidence, however, has suggested that recreation of a double coronary artery system by means of reimplantation might allow for normalization of coronary flow reserve $\mathrm{e}^{3}$ and for greater protection against secondary coronary/bypass graft changes because of age and atherosclerosis. ${ }^{2}$ On the basis of these considerations, repair by means of coronary button reimplantation was performed in our patient. Because of the unusual combination of ARCAPA and ACCAPA, translocation of the coronary button proved complex, requiring extensive skeletonization of the proximal RCA and CxA combined with the trapdoor technique, as derived by experience with the arterial switch operation. Evidence of normalization of treadmill test results 3 months after repair confirms that reimplantation should always be attempted, even in adults with isolated ARCAPA or ACCAPA.

\section{References}

1. Radke PW, Messmer BJ, Haager PK, Klues HG. Anomalous origin of the right coronary artery: preoperative and postoperative hemodynamics. Ann Thorac Surg. 1998;66:1444-9.

2. Yao CT, Wang JN, Yeh CN, Huang SC, Yang YR, Wu JM. Isolated anomalous origin of right coronary artery from the main pulmonary artery. J Card Surg. 2005;20:487-9.

3. Veselka J, Widimsky P, Kautzner J. Reimplantation of anomalous right coronary artery arising from the pulmonary trunk leading to normal coronary flow reserve late after surgery. Ann Thorac Surg. 2003;76: 1287-9.

4. Alexi-Meskishvili V, Dahnert I, Hetzer R, Lange PE, Karl TR. Origin of the circumflex coronary artery from the pulmonary artery in infants. Ann Thorac Surg. 1998;66:1406-9.

5. Bolognesi R, Alfieri O, Tsialtas D, Manca C. Surgical treatment of the left circumflex coronary artery from the pulmonary artery in an adult patient. Ann Thorac Surg. 2003;75:1642-3.

\title{
Bronchogenic cyst: Unexpected finding in a large aneurysm of the pars membranacea septi
}

\author{
Frediano Inzani, MD, ${ }^{\text {a }}$ Franco Recusani, MD, ${ }^{\mathrm{b}}$ Manuela Agozzino, MD, ${ }^{\mathrm{a}}$ Alessandra Cavallero, MD, \\ Paolo M. De Siena, MD, ${ }^{c}$ Andrea D'Armini, MD, ${ }^{c}$ Mario Viganò, ${ }^{c}$ and Eloisa Arbustini, MD, ${ }^{\text {a,d }}$ Pavia, Italy
}

I ntracardiac bronchogenic cysts are rare, with only 5 cases reported to date: 2 in the atrial septum,,${ }^{1,2} 1$ in the left atrium, ${ }^{3}$ and 2 in the right ventricle., ${ }^{4,5}$ They are identified by asymptomatic occasional findings or nonspecific symptoms, mostly in the second to fourth decades. ${ }^{3}$

Bronchogenic cysts are believed to represent a localized portion of the tracheobronchial tree that separates from the normal airways during the branching process and does not undergo further development. They develop between the 26th and the 40th day of intrauterine life, during the most active period of airway development. ${ }^{3}$ The timing of the abnormal budding may determine the location: earlier in the mediastinum and later within lung tissue, which are the 2 most common locations. ${ }^{3}$ Intracardiac location can be explained with a more precocious abnormal bud-

From the Pathology Institute, ${ }^{\mathrm{a}}$ Cardiology, ${ }^{\mathrm{b}} \mathrm{C}$ Dubost Transplant Centre, ${ }^{\mathrm{c}}$ and Transplant Research Area, Centre for Inherited Cardiovascular Diseases, ${ }^{\mathrm{d}}$ IRCCS Policlinico San Matteo, University of Pavia, Pavia, Italy.

Supported by research grants "Ricerche Finalizzate e Correnti" IRCCS Policlinico San Matteo of Pavia, Italy.

Received for publication May 31, 2006; accepted for publication June 6, 2006.

Address for reprints: Eloisa Arbustini, MD, Transplant Research Area, IRCCS Policlinico San Matteo, Piazzale Golgi 2, 27100 Pavia, Italy (E-mail: e.arbustini@smatteo.pv.it).

J Thorac Cardiovasc Surg 2006;132:972-4

$0022-5223 / \$ 32.00$

Copyright $\odot 2006$ by The American Association for Thoracic Surgery doi:10.1016/j.jtcvs.2006.06.022 ding, approximately 21 days after fertilization, when the cardiac primordial tube is near the foregut or primitive tracheobronchial tree. $^{2}$

Other noncardiac, atypical locations are cutaneous, retroperitoneal, cervical, intradiaphragmatic, intrapericardial, intraspinal, and intrapleural. $^{2}$

We report a case of a bronchogenic cyst located in the wall of a large aneurysm of the pars membranacea septi triggering premature ventricular complex (PVC) and sinus tachycardia.

\section{Clinical Summary}

A 5-year-old girl was referred to us for the occasional finding of PVC first recorded in 2001 in the third month of life, and confirmed in further controls. Two years later, a Holter monitoring documented sinus tachycardia with several PVCs. An echocardiographic study indicated a possible aneurysm of the left Valsalva sinus. The left atrium and ventricle were moderately enlarged. In 2004, a further echocardiogram described the lesion as a "subaortic aneurysm of the left ventricle."

On November 2005 the girl presented with normal cardiac tones and apparently free pauses; the chest roentgenogram showed a slightly enlarged cardiac shadow. The electrocardiogram confirmed sinus rhythm with respiratory arrhythmia and several PVCs. Transthoracic echocardiography documented an "aneurysm of the pars membranacea septi" with internal diameter of $2 \mathrm{~cm}$ (Figure 1,A). Tricuspid, mitral, aortic, and pulmonary valves showed normal function. The need for an angiocomputed tomography scan was discussed, but it was decided that echocardiography was sufficient for surgery. Indication for surgical excision was based on the potential link between the aneurysm and the PVC, and on the potential risk of emboliza- 